\title{
Bio-inspired Active Amplification in a MEMS Microphone using Feedback Computation
}

\author{
José Guerreiro, Andrew Reid, Joseph C. Jackson and James F.C. Windmill \\ Centre for Ultrasonic Engineering \\ University of Strathclyde \\ Glasgow, Scotland \\ jose.guerreiro@strath.ac.uk
}

\begin{abstract}
Auditory signal processing relies on feedback mechanisms between mechanical and electrical systems that work together to enhance acoustic conditioning. In this paper a nonlinear amplification mechanism in the mosquito's auditory system is exploited as a model of inspiration. An acoustic system that provides active amplification of sound was developed using feedback computation integrated with a MEMS microphone to implement the concept. Experimental results generated by a purpose-built embedded system show signal amplification and hysteresis which replicate the response shown by the biological mosquito's hearing system as a function of input sound intensity.
\end{abstract}

Keywords-bio-inspired acoustic sensor system; active hearing; signal amplification; feedback computation; real-time embedded signal processing; prototyping.

\section{INTRODUCTION}

Hearing capabilities are often achieved as a result of active processes that have evolved to be adaptable to cope with different environmental demands. Several auditory features have been identified which greatly enhance the ear's responsiveness in many species, humans included [1]. Amplification and tuning are two of the most important functions of a hearing organ, and therefore hearing thresholds can be at levels below the thermal vibrations of the receptor organ [2]. Considering the example of the vertebrate's inner ear, amplification is achieved due to somatic electro-motile outer hair cells acting over the basilar membrane in a sort of positive feedback mechanism pumping in additional energy that adds to the vibrational patterns inside the cochlea, providing up to 40 $60 \mathrm{~dB}$ of gain. Moreover, hearing is more sensitive to weak sounds and less responsive as signals become louder - a phenomenon called "compressive nonlinearity" [3]. This adaptive mechanism significantly increases the dynamic range achieved by a healthy hearing system; up to $120 \mathrm{~dB}$ (refer to [4] for further background knowledge about auditory sensory processing). Active hearing has also been a topic of study within other species such as insects, likewise supporting the concept that nonlinear amplification may have its origin from active cell motion inside the hearing organ [5][6]. From the engineering point of view, this sophisticated method of signal amplification and inherent tuning can provide inspiration regarding the development of adaptable sensors and systems. Designing acoustic sensors and systems that can adapt dynamically to cope with different signal demands has already been the subject of other bio-inspired studies which feature frequency agility exploited at the transducer level [7]. This paper aims to extend the topic further by presenting an unconventional signalamplifier concept with a MEMS microphone using a feedback mechanism that can provide amplification of acoustic signals at the transducer level. This faithfully mimics a nonlinear response seen in the mosquito's hearing system.

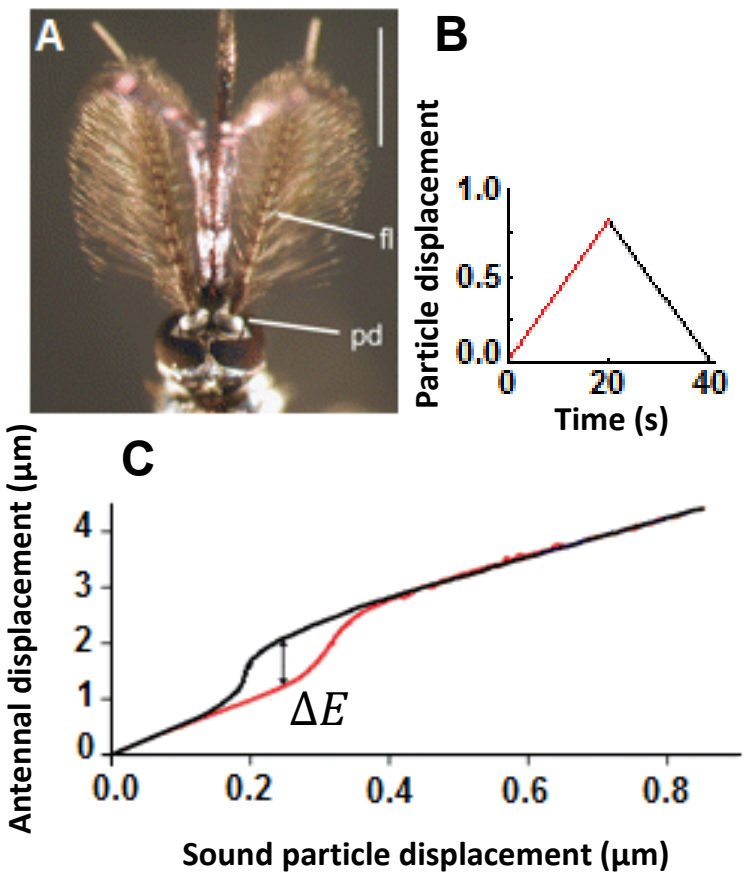

Fig. 1 -Nonlinear response of the mosquito's hearing. (A) Male antenna, highliting the plumose flagella (fl) and the pedicel (pd) - scale bar $1 \mathrm{~mm}$. (B) Envelop of the single-tone sound stimuli used to test nonlinearities in the antenna's response - increasing (red) and decreasing (black) intensity. (C) Antennal response showing amplification and hysterisis. $\Delta E$ is the rational energy of oscillation between the hysteretically amplified and the initial nonamplified response. Redrawn and adapted from [6].

\section{BIOLOGICAL SYSTEM OF INSPIRATION}

\section{A. Mosquito's Auditory System}

Mosquitoes present one of the most complex hearing systems found in insects. These antenna-like ears can detect sound through induced movement of the antennae, called a plumose flagella - a hair shaft which works as a mechanical

This research is funded by the European Research Council under the European Union's Seventh Framework Programme (FP/2007-2013) / ERC Grant Agreement n. [615030]. 
structure for sound sensing, see Fig. 1a. At the antenna base sits the pedicel in which a chordotonal organ called Johnston's organ contains up to 16,000 mechanoreceptor cells (scolopidia). These cells have the role of transducing sound induced mechanical energy into electrical signals which leads to consequent neuronal responses. Mosquitoes use their auditory receptors commonly for mating purposes. Males can detect a flying female that generates sound particle displacements of $3.5 \mathrm{~nm}$ at a distance of $10 \mathrm{~cm}$ [8], within the range of $350-450 \mathrm{~Hz}$. This ability to sense such weak and fast transient signals relies on active mechanisms with nonlinear responses that quickly amplify and tune to the signal preferentially generated from a female's beating wings.

\section{ACTIVE AMPLIFIER CONCEPT}

An initial elementary modelling of a type of acoustic receiver is to consider it as a passive oscillator. From the physical point of view, it can be approximated to a driven damped harmonic oscillator $-\mathrm{a} 2^{\text {nd }}$ order system which in Laplace form can faithfully be expressed by (1). Its resonant frequency is defined by the ratio between stiffness and mass $\left(\omega_{0}=\sqrt{\mathrm{k} / \mathrm{m}}\right)$ and quality factor expressed by the ratio between resonant frequency and damping $\left(Q=\omega_{0} / \gamma\right)$, where $k$ represents the spring factor or stiffness and $m$ is the mass of the mechanical structure.

$$
H(s)=\frac{s \frac{\omega_{0}}{Q}}{s^{2}+s \frac{\omega_{0}}{Q}+\omega_{0}{ }^{2}}
$$

A passive sensor of this type is linear with bandwidth $(\Delta w=$ $\left.\omega_{0} / Q\right)$ expressed by the ratio between its resonance frequency and $Q$ factor. On the one hand, a sensor with low-Q can achieve fast temporal resolution with the cost of a poor frequency selectivity. On the other hand, a high-Q system has a slower temporal transient responsiveness with the benefit of a higher frequency selectivity, as illustrated in Fig. 2. That sets the tradeoff within a passive acoustic sensor system and its design requirements.

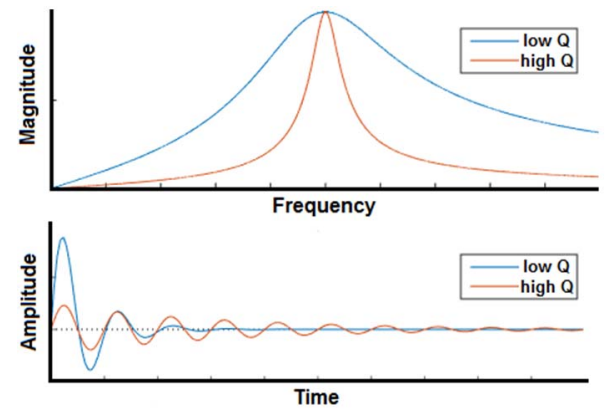

Fig. $2-\mathrm{A} 2^{\text {nd }}$ order system as the basic model for a passive acoustic sensor. Transient time response and frequency selectivity can be adapted by manipulating the $\mathrm{Q}$ factor of the system.

Importantly, for a male mosquito to sense and pursue a female successfully, its ears need to transit from a fast transient time sensing mode to a frequency-selective form of operation. That capability cannot be achieved only by a passive sensor itself. A feedback mechanism of synchronized neurons pumping additional energy to the passive mechanical system (antenna) plays the role of dynamically adapting the $\mathrm{Q}$ factor of the overall system (antenna and electro-motile neurons) when required [6]. Inspired by that unconventional mechanism of signal conditioning, an engineered system using feedback computation was developed to manipulate the response of a front-end acoustic transducer (MEMS microphone), as illustrated in Fig. 3.

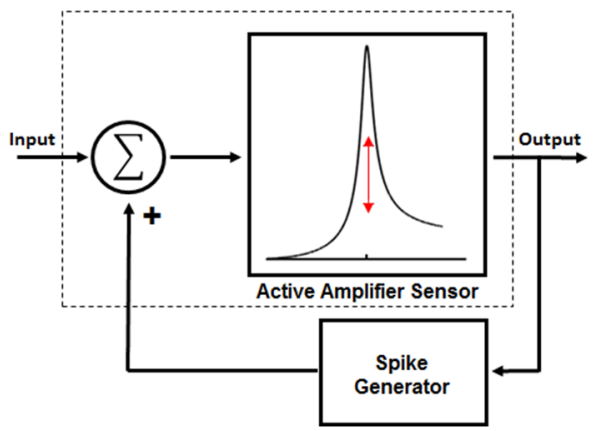

Fig. 3 - Diagram overview of the feedback control system that can be used to achieve Q control and active amplification, inspired by the mosquito's hearing system. "Active Amplifier Sensor" represents the front-end acoustic transducer (i.e. MEMS microphone); "Spike Generator" is the feedback pathway of signal computation used to pump additional energy to the front-end transducer.

\section{EMBEDDED SYSTEM SETUP}

The concept of an acoustic system with adaptive $Q$ factor that enables active amplification is engineered and an electromechanical setup is developed and presented as follows. The front-end transducer is a MEMS microphone fabricated on single crystal Silicon-On-Insulator (SOI) using MEMSCAP services (SOIMUMPs®). The device comprises an $800 \mu \mathrm{m}$ radius diaphragm mounted on 6 serpentine spring arms. The membrane itself is fringed by $176(6 \mu \mathrm{m}$ wide) capacitive combs, interdigitated with a stator bank of combs on the surrounding die at $6 \mu \mathrm{m}$ intervals, as shown in Fig. 4a. The "Spike Generator" is an embedded system computing an algorithm that generates phase-lock pulses to be entrained with the input sound signal inducing mechanical vibrations over the microphone's diaphragm (Fig. 4b).

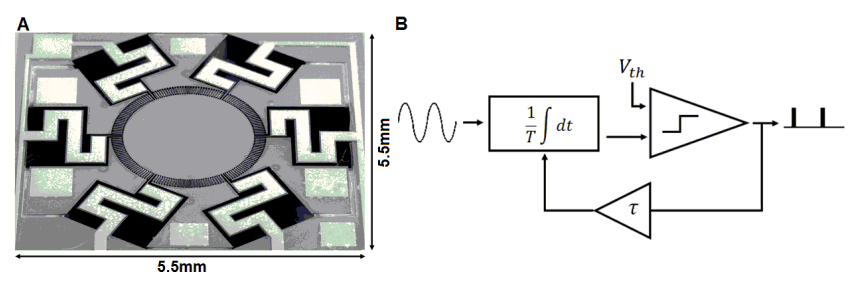

Fig. 4 - (A) SEM top view of the MEMS microphone. (B) Spike Generator simplified based on integrate-and-fire computation [9]; where $T$ represents the integrator constant, $\tau$ is the delay time to reset the integrator and $V_{t h}$ is the comparator threshold (positive value).

In order to implement a complete closed-loop system it was necessary to design an experimental setup that could provide results in real-time. The approach is based on our previous embedded system design presented in [7], with the schematic illustrated in Fig. 5. It integrates an optical readout of displacements induced by sound onto the microphone's diaphragm using a single-point laser vibrometer device 
providing correspondent analog output signals. Signal conditioning is performed on the laser output signals before $A / D$ conversion (12-bit @ $F_{s}=50 \mathrm{kHz}$ ). Signals are acquired using a STM32F4 micro-controller board running at $168 \mathrm{MHz}$. A purpose-built algorithm was developed and executed inside the processing unit that can generate spike-type phase-lock signals using a D/A converter $\left(12-b i t @ F_{s}=50 \mathrm{kHz}\right)$. The pulses can be entrained with the input signal once an average and a voltage threshold are met which thus set the system to drive the capacitive ports (capacitive combs) of the MEMS microphone. A refractory period is also defined to set the maximum firing rate of the spiking generator system. For instance, the feedback system can be adjusted to lock and fire at a specific resonant frequency which allow the $\mathrm{Q}$ factor and the overall system response to be changed, as presented in the following section.

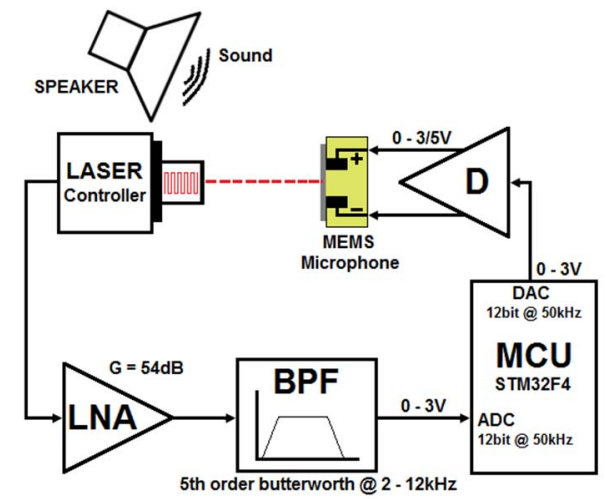

Fig. 5 - Schematic of the embedded system setup designed to provide results in real-time. Refer to [7] for additional details about circuits and systems.

\section{EXPERIMENTAL RESULTS}

Acoustico-structural interactions of the MEMS microphone were evaluated using laser Doppler vibrometer (LDV) techniques. A single-central point measurement of the microphone's diaphragm was performed during acoustic stimuli to find its natural frequency response, as illustrated in Fig. 6. One resonant frequency at $3.3 \mathrm{kHz}$ is found from a scan over the microphone's diaphragm, between $0.5-5 \mathrm{kHz}$.

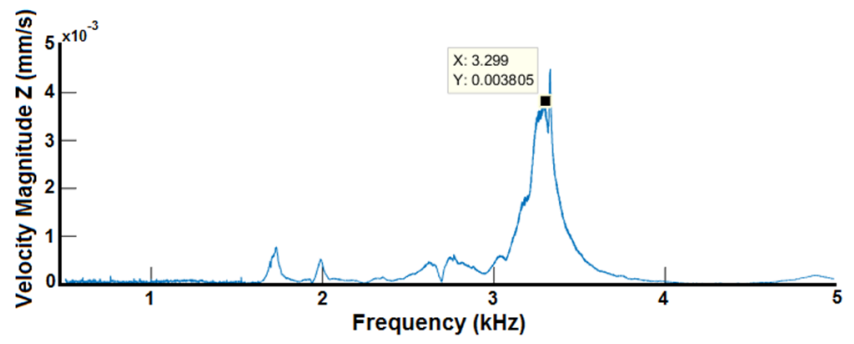

Fig. 6-Frequency response of the MEMS microphone diaphragm measured with LDV between $0.5-5 \mathrm{kHz}$.

For experimental purposes, the algorithm running on the feedback computational system was set to phase-lock and fire spike-type signals to be entrained at the resonance frequency, $3.3 \mathrm{kHz}$, of the MEMS microphone through 1:1 synchronization mode (refer to [10] for detailed background theory about synchronization and coupling between oscillators). That was engineered in order to evaluate the dynamic adaptations of the front-end transducer (passive oscillator) when coupled with a feedback system (internal oscillator) which adds extra energy to it. In Fig. 7, it is shown that without feedback entrainment of spikes, the system operates at its passive mode (see $A_{1}$ and $B_{1}$ ) with $\mathrm{Q}=30$. However, when feedback is presented through spiking activity to be entrained with the input stimuli, the sensor transits to an active mode of operation ( $\mathrm{A}_{2}$ and $\left.\mathrm{B}_{2}\right)$. It should be highlighted that at this stage the overall $\mathrm{Q}$ factor of the system is changed to $\mathrm{Q}=66$. That is indicated by the longer tail on the time domain response ( $\mathrm{A}_{2}$ red) and higher magnitude at $3.3 \mathrm{kHz}$ ( $\mathrm{B}_{2}$ red), compared with its passive mode response shown in $\mathrm{A}_{1}$ and $\mathrm{B}_{2}$, respectively. It means that the $\mathrm{Q}$ factor is essentially doubled when spiking activity is present within the system.
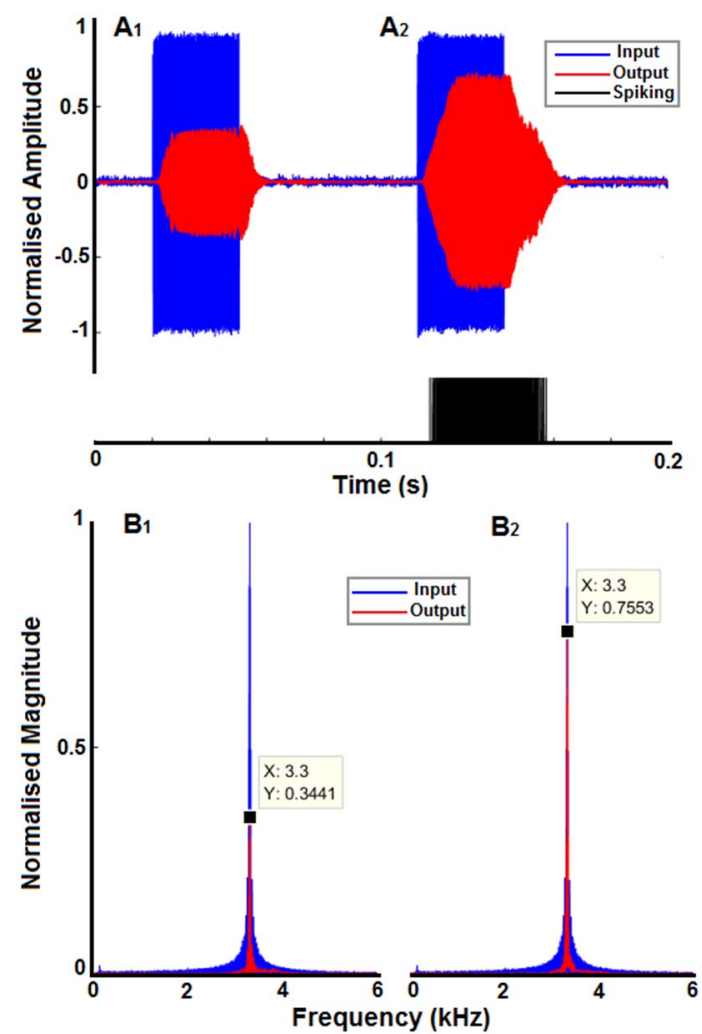

Fig. 7 - Active response of the purpose-built system. (A) Time domain single-tone acoustic stimuli at $3.3 \mathrm{kHz}$ (blue); overall response of the system (red) without-(A1) and with-(A2) phase-locked spike-type signals (black) - spike_width $=20 \mu \mathrm{s} @+5 \mathrm{~V}$. (B) Spectrum without-(B1) and with-(B2) spiking entrainment, providing a gain of around $3.4 \mathrm{~dB}$ to the overall system response.

In order to evaluate and compare the nonlinear dynamics of this engineered way of "hearing" with its biological counterpart, an amplitude modulated input signal stimuli was used in a similar manner to previous experiments with mosquitoes [6]. On the one hand, as is seen in Fig. 8, when the input signal is increased, spiking generation starts once the energy of the stimuli reaches the threshold that triggers feedback operation. On the other hand, when the stimuli is decreased, the transition from active to passive mode takes longer to happen compared with its previous state (when stimuli is increased) - that shows the hysteretic behaviour of this system as similarly seen in the mosquito's hearing system (refer to Fig. 1c for comparison). 

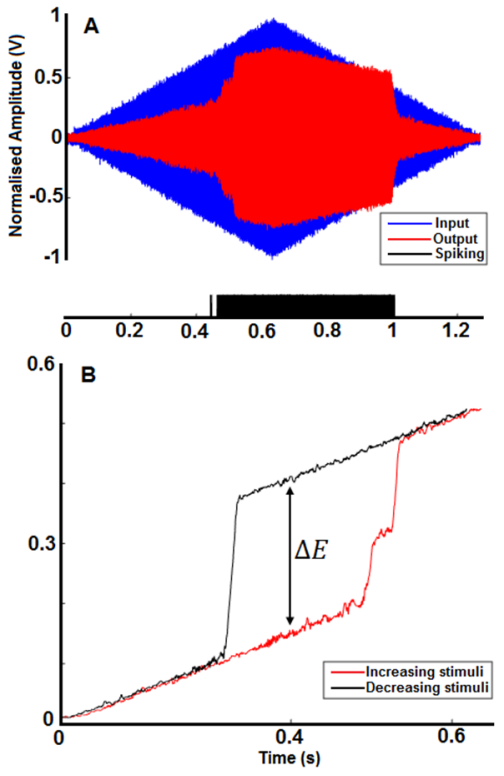

Fig. 8 - Time domain response of the purpose-built active amplifier system. (A) Single-tone acoustic stimuli at $3.3 \mathrm{kHz}$ (blue); overall response of the system (red) with phase-locked spike-type signals (black). (B) Amplification and hysteresis of the system. The rational energy $\Delta E$, represents the maximum amount of additional energy injected to the front-end sensor due to feedback computation using spike-type pulses (spike width=20 $\mathrm{s}$ @ $+3 \mathrm{~V}$ ), providing an additional energy of about $166 \%$ to the overall system response. Recall to Fig. 1c for comparison with the mosquito's nonlinear hearing response.

It should be highlighted that the entrainment between both systems (MEMS and Spike Generator) is gradual which means that at the transitory states, the spikes may not be perfectly phase-locked with the input stimuli - from that an uncontrolled behaviour of the overall system can originate. Additionally, the width, amplitude and polarity of the spikes can influence the system's stability. It also means that if the amount of additional energy injected by the feedback computation is greater than the amount of energy which can be dissipated by the front-end sensor an uncontrolled oscillatory response of the overall system can occur - behaviour that can be expressed by nonlinear active systems [10]. An additional control mechanism would ensure an improved command of the system's stability. It should also be noted that the front-end transducer consists of a MEMS microphone in its designed form, which is placed in a positive feedback loop with an internal system's generator of spike-type signals. These are short and positive square-shaped pulses (with duty-cycle smaller than $\mathrm{T} / 2$, where $\mathrm{T}$ is the period of the input signal), entrained with the input stimuli of interest. Harmonic noise that could appear at the system's output introduced by the spiking entrainment is dependent on the natural selectivity of the front-end transducer. It means that the natural frequency response of the MEMS microphone has the role of filtering out the harmonic noise resulting from the active processes within the system.

\section{CONCLUSIONS}

This paper presents a novel mechanism for sound amplification by exploiting feedback computation at the sensor level. Experimental results given by a purpose-built embedded system show dynamic adaptation of a MEMS microphone and that its Q factor can be manipulated. The overall system response shows amplification with hysteretic behaviour as similarly reported from the biological system of inspiration. This unconventional transducer concept has the potential to be applied in novel designs of sensors and systems that require dynamic adaptations in a real-time scenario. Future investigations based on the work presented in this paper might include further characterizations of the overall system response and stability while varying different parameters in the feedback loop such as, averaging time, threshold of firing, spike shape, width, amplitude and polarity. Additional study should include simulation with a theoretical model of the overall system, which can be used to better characterize the concept and understand its applicability within acoustics. Work to compare and contrast this concept with standard positive feedback amplification techniques and other techniques may also be of great interest, for example where the theory of Q control has already been studied at the circuit and system levels, such as in active analog filters [11] and atomic force microscopy [12]. It would also be valuable to investigate if the nonlinear response given by this system can be applied in a similar manner to other work that reported similar hysteretic behaviour used in speech processing [13].

\section{ACKNOWLEDGMENT}

The authors would like to thank the staff and researchers of the Centre for Ultrasonic Engineering at University of Strathclyde for all the support throughout this research work.

\section{REFERENCES}

[1] A. J. Hudspeth. "Making an Effort to Listen: Mechanical Amplification in the Ear". Neuron, 59(4): 530-545, 2008.

[2] P. Martin and A.J. Hudspeth. "Compressive nonlinearity in the hair bundle's active response to mechanical stimulation". PNAS, 98(25): 14386-14391, 2001.

[3] K. Dierkes, B. Lindner and F. Julicher. "Enhancement of sensitivity gain and frequency tuning by coupling of active hair bundles". PNAS, 105(48): 18669-18674, 2008.

[4] Schnupp, J., Nelken, I. and King, A., “Auditory Neuroscience: Making Sense of Sound". The MIT Press, 2012.

[5] M. C. Göpfert, et al., "Power gain exhibited by motile mechanosensory neurons in Drosophila ears". PNAS, 102(2): 325-330, 2005.

[6] Joseph C. Jackson, James F. C. Windmill, Victoria G. Pook and Daniel Robert, "Synchrony through twice-frequency forcing for sensitive and selective auditory processing". PNAS, 106(25): 10177-10182, 2009.

[7] José Guerreiro, Joseph C. Jackson and James F.C. Windmill. "Simple Ears Inspire Frequency Agility in an Engineered Acoustic Sensor System". IEEE Sensors Journal, 2017.

[8] J. C. Jackson and D. Robert, "Nonlinear auditory mechanism enhances female sounds for male mosquitoes". PNAS,103(45):16734-16739, 2006.

[9] A. A. Lazar, "Time encoding with an integrate-and-fire neuron with a refractory period". Neurocomputing, 58(60): 53-58, 2004.

[10] Pikovsky A., Rosenblum M., and Kurths J., "Synchronization: A Universal Concept in Nonlinear Sciences", Cambridge Univ. Press, 2001.

[11] C. D. Salthouse and R. Sarpeskar, "Jump resonance: A feedback viewpoint and adaptive circuit solution for low-power active analog filters", IEEE Trans. Circuits and Systems, 53(8): 1712-1725, 2006.

[12] Rodriguez, T. and Garcia, R., "Theory of Q control in atomic force microscopy", Applied Physics Letters, 82(26): 4821-4823, 2003.

[13] K. Aono, et al., "Exploiting jump-resonance hysteresis in silicon auditory front-ends for extracting speaker discriminative formant trajectories", IEEE Trans. Biomedical Circuits and Systems, 7(4): 389-400, 2013. 